\title{
Segmentación y clasificación de las universidades en Chile: desventajas de inicio y efectos de las políticas públicas de financiamiento
}

\author{
Segmentation and classification of universities in Chile: disadvantages \\ from the start and effects of public financing policies
}

\author{
Luis Améstica Rivas ${ }^{1} \quad$ Héctor Gaete Feres ${ }^{2} \quad$ Xavier Llinas-Audet ${ }^{3}$ \\ Recibido 28 de junio de 2013, aceptado 23 de abril de 2014 \\ Received: June 28, $2013 \quad$ Accepted: April 23, 2014
}

\begin{abstract}
RESUMEN
El presente trabajo examina cómo las políticas públicas de financiamiento destinado a las universidades estatales y privadas del Consejo de Rectores (CRUCH) en Chile han generado una segmentación económica y territorial, debido a la focalización de recursos económicos entregados históricamente a cada una de las instituciones, bajo instrumentos de financiamiento que se han mantenido por más de treinta años en el sistema, independientemente de las coaliciones y políticas gobernantes. Un análisis de correlación de variables de financiamiento actual, con datos 2011-2012, confirma las asimetrías y desigualdades entre las instituciones, especialmente para las universidades estatales de regiones, constatando sus "claras desventajas de inicio" por la entrega de recursos basales en un sistema de financiamiento a la oferta, destacando el mayor aporte otorgado a las universidades de mayor complejidad, mezclando instrumentos de financiamiento directos con subsidios a la demanda, becas y créditos, en un sistema altamente competitivo, impactando en el posicionamiento, clasificación, reputación y la percepción social sobre el prestigio de los planteles.
\end{abstract}

Palabras clave: Educación Superior, financiamiento, clasificación universidades, desigualdades y política pública.

\begin{abstract}
This paper shows to what extent a national policy for higher education state funding to finance state owned and private universities members of the Council of Rectors of Chilean Universities (CRUCH) caused economic and territorial impaired resource allocation among institutions. Despite political changes, current funding for higher education policy, implemented more than three decades ago, remains untouched. Correlation analysis of current financing variables, confirms impaired resource allocation among institutions between 2011 and 2012, specially valid for state owned regional universities, verifying "clear initial disadvantages", because of basal resource supply in an offer financing system, showing the greater amount provided to higher complexity institutions, mixing direct funding instruments with subsidies to the demand, grants and credits, in a highly competitive system, with strong effect in the ranking, classification, reputation and social perception of the institutional prestige.
\end{abstract}

Keywords: Higher Education, funding, universities ranking, inequality and public policy.

1 Facultad de Ciencias Empresariales, Universidad del Bío-Bío, Chile. E-mail: lamestica@ubiobio.cl

2 Facultad de Arquitectura, Construcción y Diseño, Universidad del Bío-Bío, Chile. E-mail: hgaete@ubiobio.cl

3 Cátedra UNESCO de Dirección Universitaria, Universidad Politécnica de Cataluña, España. E-mail: xavier.llinas@upc.edu 


\section{INTRODUCCIÓN}

Las clasificaciones en el campo de la educación superior chilena operan como un poderoso mecanismo de inclusión y exclusión de un círculo de ventajas y privilegios [1]. Estos sistemas de clasificación tienden a ser una referencia significativa en el marco del diseño de políticas públicas y reformas institucionales. La reforma educativa de los años 80 marcó el inicio de procesos de profunda privatización que constituyeron al sistema de educación superior en un mercado cuyas características principales son la ausencia de regulaciones, competencia y la liberalización de la oferta y de la demanda [2].

Los aportes financieros a las universidades desde 1981, según lo dispuesto en el Decreto Fuerza de Ley $\mathrm{N}^{\circ} 4$ de 1980 y en el artículo 2 del Decreto Supremo $\mathrm{N}^{\circ} 1783$ [3], se concentran institucional y territorialmente, generando pisos distintos entre las universidades al interior del Consejo de Rectores (CRUCH), que se traducen en "ventajas de inicio" [4] que favorecen a algunas, es decir, a políticas horizontales que incentivan la competencia están en mejores condiciones para competir y producen, obviamente, exclusión en el acceso a fondos, respaldadas en su trayectoria, capital humano, capacidad investigativa, capital social, infraestructura y madurez institucional. Estas universidades han fortalecido sus actividades y equipos humanos con el tiempo, y por eso acceden nuevamente a mayores recursos que posibilitan continuar su fortalecimiento. Asimismo, gozan de una reputación y prestigio que les permite ser selectivas, lo que repercute positivamente en la situación financiera de estas. Es decir, un círculo vicioso sustentado en una distribución histórica inequitativa, donde el financiamiento estatal basal es un factor determinante de esta segmentación.

Es sabido que la posición de las universidades en los rankings puede tener repercusiones en los subsidios que reciben del gobierno, así como en el diseño de las políticas públicas y evaluación de la educación superior. Los rankings también inciden en la elección de universidades de los estudiantes, así como en la fijación de precios o aranceles. En este contexto, los rankings internacionales están siendo cada vez más utilizados para comparar instituciones y están teniendo consecuencias en el incremento de la competencia entre las universidades, a nivel nacional y, en mayor medida, a nivel internacional entre las instituciones de más prestigio. Valle [5] establece que los rankings constituyen un elemento que forma parte del sistema de educación superior, en cuanto pueden actuar sobre las expectativas de los usuarios y las respuestas de las instituciones, cuya influencia puede llegar a modificar o intervenir en el modelo de universidad vigente.

Desde la perspectiva del financiamiento estatal a las universidades y teniendo como base el Aporte Fiscal Directo (AFD) del 95\%, fijado por el Decreto Fuerza de Ley $\mathrm{N}^{\circ} 4$ de 1980 [3], se genera una desigualdad de inicio desventajosa, especialmente para algunas universidades estatales regionales (derivadas), con lo que se marca el inicio de una clasificación y segmentación del sistema, que se mantiene hasta hoy. Al revisar estadísticamente cómo se han comportado distintos instrumentos de financiamiento dentro de las veinticinco universidades tradicionales, se constata que prevalece un mecanismo de asignación, donde la distribución porcentual del AFD del 95\% se replica y se mantiene [4]. Esto explica cómo ha evolucionado el sistema y cómo se construyen las percepciones de los agentes sobre temas de calidad, complejidad y posicionamiento.

Esta investigación pretende ser un aporte al debate en torno a la educación superior en Chile. Con datos de 2011 y 2012, aplicando el coeficiente de correlación de Pearson (R) y el de Spearman (rho), se pretende verificar el supuesto de correlación de las fuentes de financiamiento, expresadas monetariamente, de cada una de las veinticinco universidades del Consejo de Rectores (CRUCH) en el 2012, basadas en el comportamiento de los recursos del AFD del 95\% y su correspondencia con el Aporte Fiscal Directo del 5\%, Aporte Fiscal Indirecto (AFI), el Arancel de Referencia promedio ponderado por institución, las donaciones a universidades del año anterior (2011) y especialmente el último instrumento creado el 2012, denominado Aporte Basal por Desempeño.

\section{FINANCIAMIENTO UNIVERSITARIO}

Chile presenta una diversidad de instrumentos de apoyo financiero del Estado a las instituciones universitarias y a los estudiantes. Las universidades son organizaciones multiproducto [6], es decir, generan simultáneamente más de un bien: el 
conocimiento, producido mediante la investigación, y la difusión de ese conocimiento, por la enseñanza. Por lo tanto, su estructura de costo, plan de desarrollo y necesidades de financiamiento difiere entre las instituciones, es así como universidades sin fines de lucro evidencian un aumento de costos de producción por alumno [7], especialmente en la generación de carreras técnicas o con marcada presencia de laboratorios y donde coexisten niveles muy variados de complejidad académica y calidad. En este mismo sentido, las universidades son clave en la actividad científica y generan directamente más del $87 \%$ de la investigación de visibilidad internacional [8], y es evidente que el desarrollo de la actividad científica de las universidades requiere un significativo aporte financiero del Estado, como también que las estrategias de financiación estatal tienen efectos en el comportamiento de instituciones e investigadores [8-9]. Las universidades chilenas no son la excepción, y los hallazgos muestran que estos efectos podrían generar y mantener firmes brechas entre las distintas instituciones.

Chile cuenta con un complejo modelo de financiamiento de la educación superior. Se caracteriza por ser de tipo mixto -público y privado-, desde 1980, con un nivel de gasto público extremadamente bajo comparado con el gasto privado familiar [10-12]. El sistema chileno realizó una serie de cambios que incrementaron, dramáticamente, la privatización del sistema [11,13-14]. En este mismo sentido, en torno a la mitad de chilenos estudian en universidades de reciente creación [15]. El incremento de recursos públicos al sistema ha permitido que la matrícula haya llegado a una cobertura superior al 50\% de la población entre 18 y 24 años [16].

Aunque se ha alcanzado una amplia cobertura, la calidad en la formación es heterogénea [10, 17], con una oferta privada muy amplia a nivel de pregrado, gran parte de ella de dudosa calidad académica, dejando de manifiesto problemas en el nivel de certificación en algunas instituciones. Lo anterior se da en un contexto en que la calidad institucional de las carreras no está garantizada y donde el sistema de aseguramiento de la calidad ha sido cuestionado, en términos de su credibilidad [16].

La base del esquema de financiamiento es la misma que ha predominado desde 1981, basado preferentemente en los aranceles y la prestación de servicios que entregan las instituciones, con un escaso financiamiento aportado por el Estado. Con ello el régimen de autofinanciamiento permanece inalterable tal como ha acontecido en las últimas tres décadas $[11,14]$.

La política de financiamiento de los últimos años ha tendido a privilegiar, principalmente, los programas de becas y ayudas estudiantiles y, en menor medida, ampliar los fondos de tipo competitivo [18]. En este contexto, igual que en todo el mundo, las instituciones operan en un ámbito altamente competitivo, en el cual existe una gran disputa por los alumnos, por fondos de investigación y por captar el mejor personal [19]. Es así como los aranceles presentan una alta dispersión, que se mantiene a lo largo de los años, reflejando las diferencias de los objetivos institucionales, de la calidad que se imparte o las diferencias existentes en los conocimientos adquiridos por los estudiantes [20].

Las instituciones cuentan con una amplia autonomía para generar sus propios ingresos, existiendo una relativa libertad en el cobro de los aranceles. En el caso de las universidades estatales, se estima que el autofinanciamiento alcanza en promedio $74 \%$ de los ingresos totales [18]. A esto debe agregarse que existe competencia restringida debido a las asimetrías de información, presencia de regulaciones y de desigual apoyo estatal.

Las dos modalidades básicas que adopta el financiamiento estatal de la educación superior son orientadas a las instituciones (oferta) o el dirigido a los estudiantes (demanda). En varios países los recursos del Estado están ligados a criterios de desempeño y accountability. La tendencia nacional en materia de criterios de asignación de recursos públicos es, desde fines de los años noventa, a priorizar fondos concursables y financiamiento por resultados. En el contexto mundial, y así como en Chile, en los últimos años se utilizan elementos de competencia en el proceso de asignación de fondos públicos a las instituciones [9], permitiendo un apalancamiento de recursos, los que se orientan a mejorar la calidad en la formación del pregrado, postgrado y técnica, la investigación y la administración institucional.

Entre los aportes directos no concursables y de libre disponibilidad se asigna el Aporte Fiscal Directo (AFD), que en el $95 \%$ de este opera como un 
subsidio histórico exclusivo para las universidades estatales y privadas y las que se derivaron de ellas, creadas antes de 1981 [6], mientras que 5\% se hace en relación con índices de desempeño asociados. En el informe de la OCDE [12] se establece que el AFD favorece a las instituciones más grandes, más complejas y que realizan investigación más intensiva. En tanto, el Aporte Fiscal Indirecto (AFI) se asigna a todas las instituciones del sistema en virtud del número de alumnos con más altos puntajes en las pruebas de selección, direccionando los fondos públicos hacia las instituciones que tienen una alta reputación y hacia carreras de mayor valorización social. Las posibilidades de acceso y la calidad de la educación disponible para los jóvenes en Chile se asocian indisolublemente a la condición social y económica de su núcleo familiar [16].

Existe un cuantioso financiamiento a las universidades no asignable a un uso particular y que consiste en las donaciones de particulares o empresas. Desde 1998 al 2006 se ha concentrado en universidades metropolitanas y en instituciones particulares asociadas a grupos de interés religioso, económico y/o político [21]. Se observa que, en promedio, durante los últimos años (especialmente 2007 a 2010) los aportes a universidades amparados por esta ley han tenido a concentrarse en torno a una cantidad cercana a la del AFI, como ocurrió a fines de los años noventa [22]. Es evidente que las donaciones han tenido gran importancia en los avances de infraestructura de las instituciones, gracias a la propia gestión, y responden a una cierta segmentación de las universidades. En este contexto se destaca el nuevo liderazgo y los requisitos de gestión que deben enfrentar las instituciones de educación terciaria para responder mejor a las necesidades del mercado [20]. Igual que en el sistema americano, se tiende a privilegiar instituciones que ya cuentan con mayores recursos y que se orientan a segmentos más privilegiados de la población [23].

Una de las fuentes públicas más importantes del sistema son los créditos y becas, cuya asignación está determinada por los aranceles de referencia para las universidades, según una categorización, cuyo objetivo es fijar un monto máximo de ayudas para las distintas carreras de la educación superior chilena, en una base metodológica generada el 2001, y materializada en nuevas ayudas el 2005, año en que se agrupó en cuatro categorías de acuerdo con indicadores de productividad académica (investigación) y con indicadores de eficiencia en la docencia. Para su materialización se realizó una clasificación de acuerdo con componentes comunes, con el objeto de homogeneizarlas y compararlas entre sí y luego hallar al interior del grupo la de menor costo, ello hace de "referente" para el resto. Este criterio común es cuestionable que se utilice para todas las instituciones universitarias, en circunstancias de que se sabe y es legítimo que existan instituciones solo orientadas a la docencia [24].

Durante el 2012 MINEDUC (Ministerio de Educación) instauró una nueva fuente de financiamiento basada en una clasificación exclusiva para las universidades del CRUCH, llamada Aporte Basal por Desempeño; a partir de esta se segmenta en tres grupos: las de alta producción de investigación y doctorado, las de investigación focalizada en ciertas áreas y las docentes, que favorecen la movilidad social. En esta línea, Reyes y Rosso [25] señalan un esfuerzo por clasificar a las universidades según su orientación, de acuerdo con el mayor o menor acento en las funciones básicas de docencia de pre y posgrado, incluyendo el doctorado, la investigación e innovación.

\section{REPUTACIÓN Y CLASIFICACIÓN DE UNIVERSIDADES}

Todo escrutinio destinado a generar información pública representa una ventaja, en cuanto dota al interesado de elementos de juicio para decidir sus opciones de estudio y calificación futura para el mercado laboral [5]. Los agentes acuden a los rankings por diversos motivos, así los empleadores a la hora de seleccionar la procedencia de sus futuros trabajadores, los estudiantes para seleccionar donde quieren estudiar, los profesores para desarrollar su trabajo en una institución de calidad, los gobiernos tanto en cuestiones de financiación como prestigio y las propias universidades para diseñar estrategias de posicionamiento [26].

Al realizar una clasificación o rankings de universidades se suele encontrar dos líneas de trabajo que se diferencian entre sí, básicamente por su orientación metodológica [27]: las llamadas "listas reputacionales" o subjetivas, elaboradas a base de encuestas de opinión aplicadas a grupos [28]. Se caracteriza por ser un método antiguo y suele ser criticado por problemas de representatividad 
estadística, se apoyan en sondeos de imagen y reputación: evaluaciones de pares o de consumidores de servicios educativos, ya sean estudiantes, padres de familia o empleadores [28]. El segundo, son los llamados "rankings objetivos", basados en indicadores de desempeño que se calculan a partir de un determinado conjunto de datos empíricos [29], que se sustentan en procedimientos cuantitativos de evaluación, por ejemplo, la medición del número de publicaciones y citas, entre otros indicadores comparativos. Las clasificaciones del segundo tipo son los que más abundan, debido al hecho de que en internet circula un gran número de datos concernientes a variables de desempeño.

Asimismo, existen algunos que tienden a combinar ambas metodologías, es decir, indicadores cuantitativos y cualitativos [30]. Las principales críticas a estos rankings suelen referirse a que tienden a comparar realidades institucionales muy distintas $\mathrm{y}$, sobre todo, porque, excepto algunos indicadores básicos (tamaño de las poblaciones estudiantiles y académicas, recursos financieros de las instituciones, o indicadores de productividad científica), las variables de la calidad docente, o la apreciación de las funciones de difusión y extensión, suelen pasar inadvertidas [28, 31]. Entre los cuestionamientos se señalan los problemas de comparación, la selección de indicadores, la confiabilidad de la información recabada y la construcción de calificaciones unitarias en que se basa la jerarquización de las universidades. También se ha cuestionado el carácter homogeneizador de los rankings respecto de un modelo único de universidad [29, 27]. Asimismo, existen críticos de los ranking y que rechazan el concepto de universidad de rango mundial por su carácter elitista orientada a la investigación en detrimento de la docencia [32].

Albatch [33] ha señalado que los rankings son inevitables y probablemente necesarios en el competitivo mercado académico del siglo XXI, y cómo estos juegan un papel relevante en sus estrategias comerciales [29], influyendo en la selección por parte del alumnado donde cursar sus estudios. Una de las estrategia de posicionamiento en este mercado educacional por la que las universidades apuestan es la captura de buenos estudiantes [34], ya que ello conlleva, por el mecanismo de pares, al incremento del prestigio y, por lo tanto, a la atracción de nuevos buenos estudiantes. Las universidades compiten o bien por prestigio o "reputación" o bien por volumen o "cantidad de alumnos" [2]. El impacto que dentro y fuera de las instituciones están ocasionando los rankings que se publican es cada vez mayor, debido a su difusión por los medios de comunicación social. Lo importante es asegurar que provean criterios exactos y relevantes y midan las cosas adecuadas.

Una universidad que compite lo hace desplegando recursos y capacidades que son fuente de reputación o de ámbito o mediante inversión publicitaria, con distintos énfasis, acorde a su proyecto institucional [35]. En el estudio de DiGregorio y Shane [36] el prestigio está de forma considerable asociado a la calidad del plantel de profesores que pueden conformar y cómo esta impacta en las percepciones de la sociedad y les permite un mejor desarrollo de iniciativas en las áreas de investigación y desarrollo (I\&D). También los gobiernos enlazan el empleo de los rankings para basar parte de la financiación pública en los resultados de investigación o de docencia [26].

Uno de los factores determinantes en la decisión de los estudiantes para ingresar a determinada carrera universitaria es el prestigio de la universidad. El prestigio es un signo que distingue a una institución de otra, que la vuelve identificable de una manera positiva en un universo de muchos actores [37]. En tanto, Valle [5] señala que el prestigio es una categoría que se construye sobre la base de generalizaciones derivadas de percepciones públicas, pero que se indagan a partir de preguntas que el investigador define y del cruce de información que él mismo controla.

En el sistema de educación superior, una diferencia que distingue a las instituciones es el nivel de selectividad. Brunner [38] propone que esta selectividad se puede entender a partir de la diferenciación social entre instituciones, la que se efectúa en términos de sus diferencias de prestigio: las universidades de mayor e intermedia selectividad buscan controlar la expansión de su matrícula por medio de mecanismos de cierre, como la definición de altos puntajes de corte. No obstante, los rankings también pueden llegar a suponer una amenaza a la equidad[39], porque pueden limitar el acceso a los estudiantes "poco aventajados" o que sean utilizados solo por parte de los estudiantes procedentes de familias de mayores ingresos o mejor educadas. 
En los últimos años varios autores (Dill y Soo, [29]; Usher y Savino [30]; Guni [40]; Harvey [39], entre otros) han realizado críticas importantes a las metodologías utilizadas en los rankings, así como a las tipologías a las que se puede llegar. Produciendo muchas veces una percepción distorsionada del posicionamiento en los rankings con una real evaluación de la calidad de las instituciones en todos sus ámbitos de desempeño, además de crear efectos significativos en la opinión pública.

Las universidades compiten sobre la base de mejorar su reputación, buscando el prestigio social y académico de sus alumnos y profesores, la combinación de áreas de conocimiento y funciones que desarrollan, la calidad de sus servicios, el incremento de su capital social y el éxito en la generación de ingresos [38]. Se puede observar que antes las universidades tradicionales competían basadas en fuentes de reputación y prestigio académico (por ejemplo, profesores reconocidos académicamente, publicaciones, proyectos ganados), la nueva realidad del sector hace que se hayan incorporado otras dimensiones, como la calidad de la infraestructura, la presencia de profesores conocidos en su profesión, o directamente el monto de inversión publicitaria a la hora de competir en este mercado o de buscar superar las barreras a la entrada y las barreras a la movilidad para entrar en ciertos grupos más atractivos [35].

\section{CLASIFICACIÓN DE UNIVERSIDADES EN CHILE}

Las universidades chilenas difieren entre sí por el momento en que fueron creadas, su localización, tamaño, formas de propiedad, si se hallan acreditadas o no, el modo cómo combinan las funciones de docencia e investigación, sus niveles de selectividad y el origen socioeconómico de sus estudiantes. Por ejemplo, se han establecido tipologías, considerando simultáneamente si pertenecen o no al CRUCH, la propiedad, organización jurídica, tipo de bienes públicos, ingresos de titulados, por su nivel de complejidad, aporte a investigación, entre otros $[1,41-44]$.

Hasta la década de 1980 la educación superior chilena disponía de un reducido y selecto número de universidades [1], cuya constitución y misión era totalmente distinta, ya que no presentaban diferencias relevantes en cuanto a su autonomía para el desarrollo de sus funciones, su organización interna y financiamiento público [18]. Hoy esta realidad es más compleja, según lo señala la Comisión de Financiamiento Estudiantil-Mineduc [20], debido a que coexiste una variada gama de instituciones estatales y privadas, con objetivos y estructuras distintas, metropolitanas y regionales, de investigación y docencia, con y sin fines de lucro, aunque la legislación actual lo prohíbe, expresamente para las universidades [41]. Esto se traduce en la coexistencia de instituciones que, obviamente, se comportan de manera distinta, ya que tienen diferencias en sus objetivos, costos y calidad, generando con ello una suerte de competencia multidimensional.

Asimismo, declaran misiones diferentes, pueden ser religiosas o seculares, adscriben a distintos principios formativos, poseen variadas estructuras de financiamiento y modelos de negocio, y mantienen relaciones de muy diverso tipo entre sí, con la sociedad y el Estado [45]. Se observan prácticas de gestión, tradicionalmente asociadas a las empresas en la gobernanza y la dirección de las universidades con la intención de hacerlas más efectivas y eficientes [46].

En el sistema chileno parece darse el caso de una muy marcada estratificación. Brunner $[45,1]$ establece que la competencia posicional tendería a reforzar la posición de las universidades de élite, otorgando estabilidad a la cúspide de la jerarquía. Las universidades más antiguas, que han logrado un mayor capital de investigación y reputación en el tiempo, tienen obvias ventajas. No necesitan mostrarse particularmente innovadoras ni demostrar su calidad docente, lo que se reproduce automáticamente. Asimismo, la estructura de precios o aranceles asociada a las universidades de mayor prestigio refuerza la segmentación social, restringiendo las posibilidades de acceso de los que tienen menores recursos.

En contraste a lo planteado por Brunner [1], donde coloca a todas las universidades del CRUCH en una condición de privilegio y trato preferencial, esta no es tal, solo algunas han sido favorecidas. Ejemplo de ello es el aporte fiscal directo (AFD 95\%) por alumno, donde la dispersión de recursos que reciben las instituciones es significativa; durante 
el 2012 la universidad que recibió el mayor aporte fue de $\$ 1.254 .294$ y el mínimo de $\$ 82.645$ por alumno. En este mismo sentido, las instituciones de baja selectividad asumen estrategias de búsqueda de demanda generando una diferenciación social entre estudiantes. En un primer lugar, en el acceso, y en segundo lugar, según el nivel de prestigio y calidad de la institución a la que se ha ingresado. La diferenciación se produce a partir de una competencia por oportunidades escasas y distribuidas jerárquicamente para acceder a los beneficios sociales asociados al prestigio de los certificados de las universidades [45].

Otro ranking y/o clasificación a base de calidad, altamente difundido en Chile, es la acreditación institucional, existiendo coincidencia en términos de las posiciones que se asignan a cada universidad [17]. Los rankings y los procesos de acreditación presentan una relación compleja. Cumplen una función distinta; sin embargo, no pueden conducir a resultados asimétricos, ya que ambos procedimientos de evaluación deben producir información validada según criterios reconocidos y aceptados por la comunidad académica [5].

En este mismo ámbito y a partir del trabajo de clasificación de universidades, planteada por Reyes y Rosso [25], se instala el Aporte Basal por Desempeño, observando una heterogeneidad en calidad al interior de cada grupo. Se mide la calidad de las instituciones considerando la orientación de cada proyecto académico, se hace evidente que hay importantes brechas entre ellas. Estas brechas se recogen de un modo bastante imperfecto en los criterios de acreditación a nivel institucional, que constituye, por ahora, el único factor diferenciador que puede ser utilizado por los estudiantes que postulan a las universidades.

En cuanto a las clasificaciones y/o rankings de mayor difusión en Chile, son prácticamente las mismas universidades las que ocupan en uno $\mathrm{u}$ otro ranking los primeros lugares. Véase Tabla 1 , donde se sistematizan distintas clasificaciones, institucionales y/o académicas, que se han dado en el sistema en los últimos treinta años, y que han dado cuenta de una segmentación del sistema. Reconociendo los aportes de estos trabajos, los problemas que presentan algunos rankings radican en el uso de datos autorreportados, y además que estos no hacen distinción sobre la naturaleza de las instituciones o sobre su misión fundacional, complejizando la transparencia al sistema y no reconocen las diferencias de origen que han marcado su desempeño y posicionamiento actual.

\section{ANÁLISIS DE DATOS Y RESULTADOS}

La tesis a explorar radica en probar, a partir de datos monetarios oficiales correspondientes a 2011 y 2012, que existe una relación significativa de los comportamientos de los instrumentos de financiamiento (AFD del 5\%, AFI, Donaciones, Arancel de Referencia y Aporte Basal) respecto del AFD del 95\%, para ello se utilizaron los coeficientes de correlación de Pearson (R) y el de Spearman (rho), indistintamente, para verificar su validez. $\mathrm{La}$ muestra consideró las 25 universidades que reciben Aporte Fiscal Directo por parte del Estado. Aunque se trabajó con el total de las instituciones, el análisis se limita a su potencial estadístico y carácter no probabilístico.

Los resultados encontrados, cualquiera sea (véase Tabla 2), con una alta significancia, presentan una correlación positiva. Todas las fuentes de financiamiento analizadas que responden a una categorización y/o clasificación tienen como patrón de referencia la distribución inicial del Aporte Fiscal Directo del 95\%, creada en 1981. Es decir, dicho mecanismo de distribución de fondos posicionó de tal forma a las instituciones que marcó la posibilidad de captación de recursos a lo largo de treinta años de políticas públicas, por lo tanto, cualquier posibilidad de desarrollo de las instituciones más desfavorecidas está limitado por su posición inicial. El último instrumento creado, el Aporte Basal por Desempeño, en su diseño vino a replicar la segmentación histórica del sistema.

\section{De la correlación con el AFD del 5\%}

$\mathrm{Al}$ analizar la relación entre AFD del $95 \%$ y el AFD del $5 \%$ se evidencia una correlación de 0,873 y 0,837 , respectivamente, para los datos del 2012, es decir, se comportan en la misma dirección.

El AFD del 5\% es un instrumento competitivo, metodológicamente se calcula a partir de estadísticas, como son número de alumnos de pregrado, número de jornadas completas equivalentes (JCE), número de JCE con grados (Magíster o Doctor), proyectos 
Tabla 1. Criterios de segmentación y clasificación de las universidades chilenas.

\begin{tabular}{|c|c|c|c|}
\hline Ámbito & Criterio & Efectos & Origen \\
\hline Origen histórico & $\begin{array}{l}\text { Universidades antes y después del año } 81 \\
\text { (tradicionales, derivadas, privadas) }\end{array}$ & Reputación & \multirow{5}{*}{ LOCE (1981) [3] } \\
\hline $\begin{array}{l}\text { Propiedad y } \\
\text { aporte fiscal }\end{array}$ & $\begin{array}{l}\text { Universidades estatales, universidades } \\
\text { privadas con subsidio y sin aporte estatal }\end{array}$ & Segmentación & \\
\hline $\begin{array}{l}\text { Financiamiento } \\
\text { basal }\end{array}$ & Distribución Aporte Fiscal Directo (AFD) & $\begin{array}{l}\text { Segregación } \\
\text { económica }\end{array}$ & \\
\hline $\begin{array}{l}\text { Selectividad y } \\
\text { financiamiento }\end{array}$ & $\begin{array}{l}\text { Distribución Aporte Fiscal Indirecto (AFI), } \\
\text { según puntajes estudiantes }\end{array}$ & $\begin{array}{l}\text { Segregación } \\
\text { económica }\end{array}$ & \\
\hline $\begin{array}{l}\text { Localización } \\
\text { geográfica }\end{array}$ & $\begin{array}{l}\text { Universidades Región Metropolitana } \\
\text { Universidades regionales }\end{array}$ & Centro periferia & \\
\hline $\begin{array}{l}\text { Selectividad } \\
\text { académica }\end{array}$ & $\begin{array}{l}\text { Ranking de selectividad instituciones, } \\
\text { según puntajes de acceso pregrado }\end{array}$ & Posicionamiento & $\begin{array}{l}\text { Mineduc / } \\
\text { DEMRE[1-2] }\end{array}$ \\
\hline $\begin{array}{l}\text { Financiamiento } \\
\text { demanda }\end{array}$ & $\begin{array}{l}\text { Clasificación Arancel de Referencia } \\
\text { (cuatro grupos) }\end{array}$ & $\begin{array}{l}\text { Posicionamiento } \\
\text { y segmentación }\end{array}$ & $\begin{array}{l}\text { Mineduc } \\
(2001),(2005)[24]\end{array}$ \\
\hline $\begin{array}{l}\text { Enfoque ingresos } \\
\text { de egresados }\end{array}$ & $\begin{array}{l}\text { Ranking de universidades según ingresos } \\
\text { titulados }\end{array}$ & $\begin{array}{l}\text { Ranking } \\
\text { posicionamiento }\end{array}$ & $\begin{array}{l}\text { J. Benavente, D. } \\
\text { Rappoport y P. Meller } \\
(2004)[42]\end{array}$ \\
\hline Complejidad & $\begin{array}{l}\text { Universidades investigativas } \\
\text { Universidades docentes }\end{array}$ & $\begin{array}{l}\text { Ranking } \\
\text { reputación }\end{array}$ & \multirow[b]{2}{*}{ Brunner (2005) [1-2] } \\
\hline $\begin{array}{l}\text { Mercados de } \\
\text { competencia }\end{array}$ & $\begin{array}{l}\text { Clasificación a base de connotaciones } \\
\text { geográficas, distinguiendo cinco mercados } \\
\text { regionales }\end{array}$ & $\begin{array}{l}\text { Segmentación } \\
\text { reputación }\end{array}$ & \\
\hline $\begin{array}{l}\text { Selectividad } \\
\text { social }\end{array}$ & $\begin{array}{l}\text { Clasificación de instituciones por } \\
\text { origen socioeconómico de estudiantes } \\
\text { (quintilización) }\end{array}$ & $\begin{array}{l}\text { Segmentación } \\
\text { posicionamiento }\end{array}$ & $\begin{array}{l}\text { Mineduc (2006) } \\
{[1-2]}\end{array}$ \\
\hline $\begin{array}{l}\text { Calidad } \\
\text { institucional }\end{array}$ & Clasificación por años de acreditación & $\begin{array}{l}\text { Ranking } \\
\text { posicionamiento }\end{array}$ & CNA (2006) $[1-2,47]$ \\
\hline $\begin{array}{l}\text { Funciones } \\
\text { y resultados }\end{array}$ & $\begin{array}{l}\text { Clasificación en ocho categorías de } \\
\text { universidades en el sistema }\end{array}$ & $\begin{array}{l}\text { Categorización de } \\
\text { universidades }\end{array}$ & Bernasconi (2006) [1] \\
\hline $\begin{array}{l}\text { Universidades } \\
\text { investigadoras }\end{array}$ & Índice -H de universidades chilenas & $\begin{array}{l}\text { Ranking de } \\
\text { prestigio }\end{array}$ & Rau, J. (2008) [44] \\
\hline $\begin{array}{l}\text { Funcionalidad y } \\
\text { complejidad }\end{array}$ & $\begin{array}{l}\text { Tipología a base de siete grupos distintivos } \\
\text { de universidades en el sistema chileno }\end{array}$ & $\begin{array}{l}\text { Posicionamiento y } \\
\text { reputación }\end{array}$ & Brunner (2009) [1] \\
\hline $\begin{array}{l}\text { Bienes } \\
\text { producidos }\end{array}$ & $\begin{array}{l}\text { Clasificación en virtud de los bienes } \\
\text { públicos que entregan a la sociedad }\end{array}$ & $\begin{array}{l}\text { Posicionamiento y } \\
\text { reputación }\end{array}$ & Parada, J. (2010) [41] \\
\hline $\begin{array}{l}\text { Tipo de público al } \\
\text { que atienden }\end{array}$ & $\begin{array}{l}\text { Clasificación de universidades por } \\
\text { selectividad y función (cuatro criterios) }\end{array}$ & $\begin{array}{l}\text { Ranking } \\
\text { posicionamiento }\end{array}$ & $\begin{array}{l}\text { Torres y Zenteno. } \\
\text { (2011) [43] }\end{array}$ \\
\hline $\begin{array}{l}\text { Líderes en } \\
\text { investigación }\end{array}$ & $\begin{array}{l}\text { Ranking Universidades del CRUCH en } \\
\text { SIR (SCImago Institutions Ranking) }\end{array}$ & $\begin{array}{l}\text { Ranking } \\
\text { posicionamiento }\end{array}$ & $\begin{array}{l}\text { Ramírez, P. y Alfaro, } \\
\text { L.(2012) [8] }\end{array}$ \\
\hline $\begin{array}{l}\text { Investigación o } \\
\text { docencia }\end{array}$ & Ranking según su orientación institucional & $\begin{array}{l}\text { Posicionamiento } \\
\text { y segmentación }\end{array}$ & $\begin{array}{l}\text { Reyes y Rosso (2012) } \\
{[25]}\end{array}$ \\
\hline $\begin{array}{l}\text { Financiamiento } \\
\text { oferta }\end{array}$ & $\begin{array}{l}\text { Clasificación Aporte Basal Desempeño } \\
\text { (tres grupos) }\end{array}$ & $\begin{array}{l}\text { Posicionamiento y } \\
\text { segmentación }\end{array}$ & $\begin{array}{l}\text { Mineduc } \\
(2012)[25]\end{array}$ \\
\hline $\begin{array}{l}\text { Enfoque recursos } \\
\text { estratégicos }\end{array}$ & $\begin{array}{l}\text { Composición de diez grupos estratégicos } \\
\text { homogéneos de universidades chilenas }\end{array}$ & $\begin{array}{l}\text { Ranking de } \\
\text { reputación y } \\
\text { posicionamiento }\end{array}$ & $\begin{array}{l}\text { Thieme, J., Araya- } \\
\text { Castillo, L. y } \\
\text { Olavarrieta, S. (2012) } \\
\text { [35] }\end{array}$ \\
\hline
\end{tabular}

Fuente: Elaboración propia. 
Tabla 2. Correlaciones AFD del 95\%.

\begin{tabular}{|l|c|c|c|c|c|c|}
\hline \multicolumn{2}{|c|}{} & AFD del 5\% & AFI & Donaciones & $\begin{array}{c}\text { Arancel } \\
\text { Referencia }\end{array}$ & $\begin{array}{c}\text { Aporte } \\
\text { Basal }\end{array}$ \\
\hline \multirow{3}{*}{$\begin{array}{l}\text { Correlación } \\
\text { de Pearson }\end{array}$} & Coeficiente & 0,873 & 0,939 & 0,838 & 0,818 & 0,728 \\
\cline { 2 - 7 } & Sig. (bilateral) & 0,000 & 0,000 & 0,000 & 0,000 & 0,000 \\
\cline { 2 - 7 } & $\mathrm{N}$ & 25 & 25 & 25 & 25 & 25 \\
\hline \multirow{2}{*}{$\begin{array}{l}\text { Rho de } \\
\text { Spearman }\end{array}$} & Coeficiente & 0,837 & 0,809 & 0,557 & 0,809 & 0,764 \\
\cline { 2 - 7 } & Sig. (bilateral) & 0,000 & 0,000 & 0,004 & 0,000 & 0,000 \\
\cline { 2 - 7 } & $\mathrm{N}$ & 25 & 25 & 25 & 25 & 25 \\
\hline
\end{tabular}

Fuente: Elaboración propia a base de datos Mineduc.

de investigación y publicaciones. Aunque se le ha asignado un efecto recursivo al AFD, especialmente para la universidades investigativas, lo que afecta el crecimiento o decremento del monto recibido del AFD del 5\% para algunas instituciones [8]. Lo que es evidente que estos recursos se entregan en relación con el desempeño histórico del plantel, y que tienen una alta correlación con índices de productividad, que han sido fruto de un fortalecimiento institucional que se ha venido construyendo con la historia, favoreciendo a quienes están en mejores condiciones para competir, generando exclusión y concentración [4].

\section{De la correlación con el AFI}

$\mathrm{Al}$ analizar estadísticamente la relación entre AFD del $95 \%$ y el AFI se constata la desigualdad de inicio, evidenciando una correlación de 0,934 y 0,809 para los indicadores propuestos, dentro de las veinticinco universidades del CRUCH para el 2012, es decir, se comportan en forma similar.

Como se señaló, el AFI es instrumento basado en una clasificación de selectividad, fue pensado para promover calidad fomentando la competencia por los mejores estudiantes; se asigna de acuerdo con una escala de puntajes de admisión de los estudiantes, con aportes diferenciados en cinco tramos, siendo el tramo de puntajes más altos el que recibe más recursos. Las universidades del CRUCH captaron durante el 2012 \$ 17.100 millones del total entregado al sistema [47], donde su captación está de forma sólida vinculada con las percepciones de calidad de los estudiantes, en especial respecto de las universidades más prestigiosas y la valorización social de las carreras. Es así como cinco universidades concentran el $77 \%$ de lo captado por las veinticinco instituciones del país, representando el 39\% de la matrícula total del CRUCH. Por esta razón, la captación de los recursos se ha mantenido estable y concentrada en un pequeño grupo de instituciones.

Al existir una clara conexión entre el nivel socioeconómico de los alumnos y sus resultados en las pruebas de selección, la operación del AFI es socialmente regresiva, y es análogo a la alta desigualdad y segregación que presenta el sistema universitario chileno. En un trabajo anterior, Wörner [34] había hecho presente esta característica. En efecto, según antecedentes provistos por el MINEDUC[47], alrededor de $42 \%$ de los alumnos que obtiene AFI proviene de colegios privados pagados, $58 \%$ proviene de colegios subvencionados y municipalizados, con una concentración del $55 \%$ en la Región Metropolitana. Se observa un direccionamiento de la financiación pública a las instituciones con alta reputación, cuyos estudiantes, es más probable, provienen de familias más pudientes $[12,34]$.

\section{De la correlación con las donaciones a universidades}

Al revisar la relación entre el AFD del 95\%, donde prevalece un mecanismo de asignación histórica y las donaciones privadas, evidenciamos una correlación de 0,838 y 0,557 , indistintamente para cada uno de los parámetros utilizados, para datos del 2011. Es decir, se comportan en forma similar, las universidades con mayor AFD del $95 \%$ obtienen mayores donaciones.

Entre 2001 y 2011 el sistema universitario en su conjunto recibió $\$ 191$ mil millones de pesos gracias a la ley de donaciones. Vargas y Martínez [22] 
establecieron que el $46 \%$ del total se concentraron en solo dos planteles: ambas instituciones privadas católicas y enfocadas a la formación de jóvenes de altos ingresos; $y$ de las diez instituciones que captan la mayor cantidad de recursos, ninguna figura entre las diez instituciones que atienden a los sectores de menor nivel socioeconómico del país. Asimismo, se evidencia una gran focalización de recursos hacia instituciones privadas frente a las públicas, inclusive dentro del CRUCH, con una alta concentración en la Región Metropolitana, significando el 76,3\% de las donaciones país [21]. Entre las diez instituciones con mayores donaciones del sistema solamente la Universidad de Chile es estatal. Del resto, tres son universidades privadas pertenecientes al $\mathrm{CRUCH}$ y seis son privadas no tradicionales.

El acceso a recursos donativos que distintas universidades pueden alcanzar se traduce en mejoras de infraestructura, en calidad de servicios, beneficios pecuniarios para los miembros del plantel de académicos, o para los dueños del capital de cada organización, en el caso de las universidades privadas, traduciéndose en ventajas de posicionamiento atribuibles a la influencia: factores como redes sociales, grupos de interés, políticos, económicos, núcleos empresariales, religiosos, entre otros, que tienen como elementos determinantes el estatus social. Como lo señalan Vargas y Martínez [22], la mayor parte de los donantes del sistema corresponde a grupos empresariales de origen nacional, de tipo familiar, que hacen aportes permanentes o frecuentes al sistema universitario, tanto a título personal como desde las empresas que controlan.

La ley ha servido como un instrumento que le entrega a los privados la facultad de decidir a quién beneficia. Y como una parte de estos dineros provienen de una reducción de impuestos, al final de la cadena. El Fisco contribuye económicamente al mismo fin que el privado determina $36 \%$ de este monto correspondió a recaudación fiscal no percibida por el Estado [22], la que pasó a ser asignada discrecionalmente por determinados contribuyentes.

En este sentido, muchas veces las donaciones suponen la afiliación de una universidad con una organización socialmente reconocida. Ejemplo de ello son algunas universidades privadas en el mundo creadas por una organización religiosa, una empresa comercial, una fundación filantrópica, cuyo alcance se extiende más allá de la educación, y para los que la universidad no es más que uno de los medios a su disposición para promover sus misiones [48].

\section{De la correlación con el Arancel de Referencia Promedio Ponderado por institución}

Para realizar esta asociación previamente se realizaron algunos ajustes metodólogos que permitieran comparar las universidades. Se obtuvo con datos del 2012 un arancel de referencia promedio por institución a partir de cada carrera, pero debidamente ponderado por vacantes ofrecidas, circunscritos a programas de pregrado mayores de ocho semestres académicos y que se ofrecen solo por admisión PSU. De esta forma se eliminó la inconsistencia en el valor de los aranceles de programas de continuación de estudios vespertino y de carácter tecnológico.

Estadísticamente se correlacionó el AFD del 95\% con el Arancel referencial (AR) promedio ponderado por institución, evidenciando una correlación positiva para ambos parámetros, Pearson de 0,818 , y de Spearman 0,809. Análogo a los análisis anteriores, también se comporta en forma similar, las universidades con mayor AFD del 95\% presentan aranceles de referencia (AR) mayores para financiar becas y créditos de los estudiantes de pregrado.

Es sabido que la actual metodología utilizada para el cálculo de los AR es compleja, ya que involucra múltiples etapas y un gran volumen de cálculos. Así, para obtener el monto del reajuste de un determinado AR se deben agrupar las carreras entre carreras similares, para luego ser clasificadas en función del desempeño y los años de acreditación de las instituciones. Bajo la actual metodología de AR los indicadores de desempeño académico (número JCE con magister y doctorados, el número de proyectos Fondecyt y Fondef y el número de publicaciones ISI y Scielo) determinan más del $60 \%$ del resultado de desempeño institucional de una universidad [24]. Es decir, a las universidades mejor categorizadas se les fijó un mayor arancel que al resto.

Debido a la clasificación de las universidades se observa una segmentación clara. El 97\% de los indicadores de eficiencia docente se construye a base de la tasa de retención, lo que se transforma en un problema para aquellas universidades que concentran mayor proporción de alumnos vulnerables, ya que 
esta variable está altamente correlacionada con el nivel socioeconómico del estudiante [24].

\section{De la correlación con el Aporte Basal por Desempeño}

El contenido recursivo del instrumento se constata al comparar la distribución del AFD del 95\%, con la propuesta de distribución del Aporte Basal por Desempeño hecha por el MINEDUC en diciembre del 2012. Comprobamos la alta correlación que existe entre ambos instrumentos, con 31 años de desfase en la concepción de política pública. En este sentido, tanto el diagrama de dispersión como los coeficientes de Pearson $(0,728)$ y el de Spearman $(0,764)$ evidencian distribuciones de los aportes por institución que tienden a ser similares.

En definitiva, el nuevo instrumento consolida las antiguas "ventajas de inicio", siguiendo un path dependence que da cuenta de una acumulación de competencias a lo largo del tiempo.

Aunque en su génesis la "clasificación horizontal" no debe operar como un ordenamiento vertical, en términos prácticos, esta agrupación genera una señal indirecta a los actores relevantes y que ubica equivocadamente a las universidades como de "primera, segunda y tercera división". Los criterios utilizados y las etiquetas para cada categoría hacen que esta clasificación se asimile por los actores como un ranking, cuestión que se reafirma por lo asimétrico de la distribución de los recursos financieros donde la primera universidad tiene 5,6 veces más que la última universidad.

Desde el punto de vista metodológico, cualquiera sea la clasificación de las universidades, las variables utilizadas pueden ser consideradas como discrecionales, inclusive de un análisis estadístico podrían surgir más o menos categorías, dependiendo por tanto de una decisión política que permita reflejar la compleja realidad del sistema universitario.

\section{CONCLUSIONES}

En Chile, el financiamiento vía el AFD del 95\% para las universidades constituye un aporte permanente que contribuye al financiamiento de una masa crítica con dedicación a docencia e investigación, infraestructura, vinculación con el medio, entre otros. $\mathrm{Al}$ existir una alta dispersión en los porcentajes de distribución del AFD del 95\% entre las instituciones del CRUCH, eso se traduce que las de mayor aporte, mantienen una mayor relación con la contribución que estas realizan en el ámbito de todas sus tareas nobles, especialmente en investigación y desarrollo. Por lo tanto, algunas instituciones destacan por la acumulación de patrimonio a lo largo del tiempo, y con ello mantienen y fortalecen su posición en cualquier ranking o clasificación que mida su desempeño académico. Ser o no una institución selectiva en Chile responde en parte a la tradición y en parte a la naturaleza de su misión fundacional y a la política de financiamiento.

Como se evidenció, en el nivel medio bajo de desarrollo se ubican algunas de las universidades derivadas de regiones, que a pesar de sus esfuerzos necesitarán muchos años más para alcanzar el nivel de excelencia de las que han tenido las instituciones de mayor aporte de AFD del 95\%. Por lo tanto, existe una deuda histórica con estas universidades, y cuya reivindicación es necesaria si se quiere tener un país más justo e igualitario. Lo anterior no supone una "estrategia tipo Robin Hood", es decir, quitarle a lo más ricos para darle a los pobres, sino más bien reconocer esta brecha y fortalecer a las más desventajadas.

Es evidente la importancia de las clasificaciones y rankings para un buen funcionamiento del sistema, en particular para una correcta elección de parte de los padres, estudiantes, empleadores, académicos, sobre instituciones y programas es la información disponible. Los esfuerzos que ha realizado la autoridad con el fin de aumentar la información son valiosos, pero persisten importantes asimetrías de información que son difíciles de atenuar.

Los mecanismos habituales de las clasificaciones revisadas surgen a partir de un análisis de "cluster estadísticos" y en forma posterior a un estudio de los componentes principales que permite agrupar las variables más explicativas, generando un potencial problema de "colinearidad", generando agrupamientos a partir de indicadores altamente correlacionados, que se mueven en un mismo y único ámbito de desarrollo y de desempeño institucional.

Lo anterior ayuda a explicar cómo se mueven las fuentes de financiamiento, producto de las variables utilizadas en las clasificaciones actuales, y que por 
ende han afectado la distribución de recursos entre las instituciones, relacionándose con los recursos entregados históricamente por medio del AFD del $95 \%$, al vincular esta clasificación con la inversión pública, orientada a estimular un desarrollo más equilibrado y armónico, generando un marco que favorece las "ventajas de inicio" de cada una de las instituciones, las que son producto de una acumulación histórica de apuestas discrecionales de parte del Estado.

Con este estudio se ha colocado en el tapete el financiamiento de las universidades en Chile. Sin duda, persisten interrogantes respecto de los factores explicativos que podrían constituir materia de futuras investigaciones, que incorporen un análisis factorial basado en una metodología de componentes principales.

\section{REFERENCIAS}

[1] J.J. Brunner. "Sobre la clasificación de universidades". Pensamiento Educativo: Revista de Investigación Educacional Latinoamericana. Vol. $50 \mathrm{~N}^{\circ}$ 1, pp. 115-129. Apr. 2013.

[2] J.J. Brunner y D. Uribe. "Mercados universitarios: el nuevo escenario de la educación superior". Universidad Diego Portales. Santiago, Chile. 2007.

[3] Consejo de Rectores Universidades Chilenas. "Normas generales de la Educación Superior". Régimen jurídico la Edución Superior. Vol. 1, pp. 19-27. 1982.

[4] H. Gaete. "21 años de distribución centralista: el Aporte Fiscal Directo a las Universidades". Política Sectorial, Asuntos Públicos - CED. Vol. 226. 2002.

[5] A. Valle. "Idoneidad de los rankings universitarios". Revista Calidad en la Educación. Vol. 25, pp. 109-126. 2006.

[6] C. Williamson y J.M. Sánchez. "Financiamiento universitario : principios básicos para el diseño de una politíca pública en Chile". Temas Agenda Pública, PUC. Vol. 34, pp. 1-30. 2009.

[7] D. Laband and B. Lentz. "Do Costs Differ Between For-Profit and Not-for-Profit Producers of Higher Education?". Research in Higher Education. Vol. 45, Issue 4, pp. 429441. June, 2004.
[8] P.E. Ramírez y J.L. Alfaro. "Desincentivo a la Investigación: Resultado del Comportamiento Inequitativo del Modelo de Aporte Fiscal Directo (AFD) a las Universidades Chilenas". Formación Universitaria. Vol. $5 \mathrm{~N}^{\circ} 4$, pp. 27-36. 2012.

[9] I. Liefner. "Funding, resource allocation, and performance in higher". Higher Education. Vol. 46, Issue 4, pp. 469-489. 2003.

[10] R. Schmal, R. Ruiz, S. Donoso and M. Schaffernicht. "Factores que inciden en el financiamiento de los estudios universitarios en Chile”. Sociologías. Vol. 9 N$^{\circ} 17$, pp. 316339. 2007.

[11] S. Donoso. "Políticas e instrumentos de financiación de estudios universitarios: encrucijadas para el diseño de sistemas de financiamiento sustentables". Ensaio: Ens. aval. pol. públ. Vol. 16 N 60, pp. 359-392. 2008.

[12] OECD. "Tertiary Education in Chile". Reviews of National Policies Education. Paris, Francia. 2009.

[13] O. Espinoza. "Creating (in) equalities in access to higher education in the context of structural adjustment and post-adjustment policies: the case of Chile". Higher Education. Vol. 55, Issue 3, pp. 269-284. February, 2007.

[14] S. Donoso. "Economía política del financiamiento de los estudios universitarios en Chile (1980-2010): debate de sus fundamentos". Innovar. Especial en Educación, pp. 141-156. Diciembre 2009.

[15] J. Barandiaran. "Threats and opportunities of science at a for-profit university in Chile". Higher Education. Vol. 63, Issue 2, pp. 205218. April, 2011.

[16] E. Rodríguez-Ponce. "La educación superior en Chile y el rol del mercado: ¿culpable o inocente? Ingeniare. Revista chilena de ingeniería. Vol. $20 \mathrm{~N}^{\circ}$ 1, pp. 126-135. 2012.

[17] O. Espinoza and L.E. González. "Accreditation in higher education in Chile: results and consequences". Quality Assurance in Education. Vol. 21, Issue 1, pp. 20-38. 2013.

[18] G. Zapata, I. Tejeda y A. Rojas. "Educación Superior en Chile - Informe Nacional". CINDA Educación Superior en Ibero América. 2011.

[19] X. Llinás-Audet, M.Girotto and F. Solé Parellada. "La dirección estratégica 
universitaria y la eficacia de las herramientas de gestión: el caso de las universidades españolas". Revista de Educación. Vol. 355, pp. 33-54. 2011.

[20] Comisión de Financiamiento Estudiantil para la Educación Superior. "Análisis y recomendaciones para el sistema de financiamiento estudiantil". Mineduc. Santiago, Chile. 2012.

[21] L. Améstica."A.T.I., El Aporte Fiscal que no se ve". Documento de trabajo, Programa Políticas Públicas-UBB, pp. 1-21. 2007.

[22] E. Vargas y C. Martínez. "Evaluación del sistema de franquicias tributarias para donaciones a universidades en chile". Trabajos de Investigación en Políticas Públicas, U. Chile. Vol. 12, pp. 1-18. 2012.

[23] M. Yetman and R. Yetman. "How does the Incentive of the Charitable Deduction Vary Across Charities?". Accounting Review. Vol. 88, Issue 3, pp. 1069-1094. May, 2013.

[24] L. Escobar, J.M. Eguiguren y R. Sánchez. "Aranceles de Referencia de la Educación Superior. Estado actual, metodología de cálculo y desafíos". Dirección de Presupuesto. Santiago, Chile. 2012.

[25] C. Reyes y P. Rosso. "Una nueva clasificación de las universidades chilenas". Documento de Trabajo Red Universitaria Cruz del Sur. Santiago, Chile. 2012.

[26] M. Thakur. "The impact of ranking systems on Higher Education and its stakeholders". Journal of Institutional Research. Vol. 13, Issue 1, pp. 83-96. 2008.

[27] I. Ordorika and R.R. Gómez. "El ranking Times en el mercado del prestigio universitario". Perfiles Educativos. Vol. XXXII, $\mathrm{N}^{\circ} 129$, pp. 8-29. 2010.

[28] G. Federkeil. "Rankings and Quality Assurance in Higher Education". Higher Education in Europe. Vol. 33, Issue 2-3, pp. 219-231. 2008.

[29] D. Dill and M. Soo. "Academic quality, league tables, and public policy: A crossnational analysis of university ranking systems". Higher Education. Vol. 49, Issue 4, pp. 495-533. 2005.

[30] A. Usher and M. Savino. "A Global Survey of University Ranking and League Tables" Higher Education in Europe. Vol. 32, Issue 1, pp. 5-15. 2007.
[31] M. Ishikawa. "University Rankings, Global Models, and Emerging Hegemony: Critical analysis from Japan". Journal of Studies Interantional Education. Vol. 13, Issue 2, pp. 159-173. 2009.

[32] D. Hendel and I. Stolz. "A comparative analysis of higher ranking systems in Europe". Tertiary Education and Management. Vol. 14, Issue 3, pp. 173-189. 2008.

[33] P. Altbach. "International higher education, reflections on policy and practice". Boston College CIHE. Boston, USA. 2006.

[34] C.H. Wörner. "The pursuit of prestige: distribution of talented students in Chile's Universities". Avaliação. Vol. 16, Issue 2, pp. 463-476. 2011.

[35] J. Thieme, L. Araya-Castillo y S. Olavarrieta. "Grupos estratégicos de universidades y su relación con el desempeño: el caso de Chile". Innovar. Vol. $22 \mathrm{~N}^{\circ}$ 43, pp. 105-116. 2012.

[36] D. Di Gregorio and S. Shane. "Why do some universities generate more start - ups than others?". Research Policy. Vol. 32, Issue 2, special, pp. 209-227. February, 2003.

[37] P. Cyrenne and H. Grant. "University decision making o and prestige: An empirical study". Economics of Education Review. Vol. 28, Issue 2, pp. 237-248. April, 2009.

[38] J.J. Brunner. "Educación superior en Chile. Instituciones, mercados y políticas gubernamentales (1967-2007)". Doctoral thesis. Leiden University. 2008.

[39] L. Harvey. "Ranking of Higher Education Institutions: A Critical Review!". Quality Higher Education. Vol. 14, Issue 3, pp. 187 207. 2008.

[40] GUNI. "La Educación Superior en el mundo 2007”. Ed. Mundi-prensa. Madrid, España. 2007.

[41] J.R. Parada. "Universidades Públicas y privadas: Un enfoque tridimensional". Estudios Públicos. Vol. 120, pp. 183-205. 2010.

[42] J. Benavente, D. Rappoport y P. Meller. "Rankings de universidades chilenas según los ingresos de sus titulados". Documento de Trabajo. Banco Central de Chile. Vol. 306, pp. 1-42. 2004.

[43] R. Torres y M. Zenteno. "Sistema de educación superior: Una mirada desde las instituciones y sus características". Foro de Educación Superior AEQUALIS, pp. 19-77. Santiago, Chile. 2011. 
[44] J. Rau. "Índice-h de universidades chilenas líderes en investigación y su relación con rankings basados en la percepción de su prestigio". Información, Cultura y Sociedad. Vol. 18, pp. 77-84. 2008.

[45] J.J. Brunner. "El sistema de educación superior en Chile: un enfoque de economía política comparada". Avaliação. Vol. $13 \mathrm{~N}^{\circ}$ 2, pp. 451-486. 2008.

[46] A. Bernasconi. "Constitutional prospects for the implementation of funding and governance reforms in Latin American higher education". Journal of Education Policy. Vol. 22, Issue 5, pp. 509-529. September, 2007.

[47] MINEDUC. Servicio de Información de Educación superior (SIES). 2012.

[48] A. Bernasconi. "Does the Affiliation of Universities to External Organizations Foster Diversity in Private Higher Education? Chile in Comparative Perspective". Higher Education. Vol. 52, Issue 2, pp. 303-342. September, 2006. 